\title{
REVIEW
}

\section{COMBINATION IMMUNOTHERAPY IN METASTATIC RENAL CELL CARCINOMA}

\author{
P. Sepe, A. Ottini, E. Verzoni, V. Guadalupi, M. Claps, G. Procopio \\ Department of Medical Oncology Unit, Fondazione IRCCS Istituto Nazionale dei Tumori di Milano, Milan, Italy
}

\section{CORRESPONDING AUTHOR:}

Pierangela Sepe

Department of Medical Oncology Unit

Fondazione IRCCS Istituto Nazionale dei Tumori di Milano

via G. Venezian 1

20133 Milan, Italy

E-mail: pierangela.sepe@istitutotumori.mi.it

Doi: $10.48286 / a r o .2021 .10$

History

Received: Jan 9, 2021

Accepted: Feb 12, 2021

Published: Mar 1, 2021

\begin{abstract}
Various treatments have been considered as the cornerstone for the management of patients with metastatic renal cell carcinoma (mRCC) over the past two decades. Currently, immunotherapy is a promising clue in the landscape of frontline treatment of mRCC. Immune checkpoint inhibitors (ICls), which constitute a standard therapy in pretreated mRCC patients, are emerging as possible earlier treatment's strategy in mRCC. Otherwise, antiangiogenetics are well established as a backbone therapy for mRCC, and research is now focused on development of innovative tyrosine kinase inhibitors (TKIs). Frontline combination with
\end{abstract}

ICls as well as strategies including both TKIs and immunotherapy demonstrated to significantly improve outcomes compared to single-agent antiangiogenetics. Nonetheless, a considerable proportion of patients shows primary resistance to $\mathrm{ICls}$ and new approaches are currently emerging to resolve this important unmet need. Morover, several treatment strategies combining different mechanisms of action or targeting immune escape pathways are rising with the objective of improving response rates and patient's outcomes. This review summarizes current immunotherapeutic agents approved for mRCC.

\section{KEY WORDS}

Metastatic renal cell carcinoma; immunotherapy; anti-PD-1; anti-CTLA-4; targeted therapy; biomarkers. 


\section{INTRODUCTION}

Kidney cancer has peculiar features that make it attractive for immunotherapeutic approaches: chemoresistance and immunogenicity (1). Angiogenesis and immunosuppression play a relevant role in metastatic renal cell carcinoma (mRCC) carcinogenesis. Over the last two decades, different therapies including angiogenesis inhibitor monoclonal antibodies, multitarget molecules such as vascular endothelial growth factor receptor (VEGFR) inhibitors, and other tyrosine kinase inhibitors (TKIs), as well mammalian target of rapamycin (mTOR) inhibitors, have been considered the backbone for the treatment of MRCC (2-7). On the other hand, a new therapeutic approach has opened up in the frontline setting of treatment with immunotherapy $(8,9)$.

Immune checkpoint inhibitors (ICl's) already represent a well know treatment option in pretreated mRCC patients, and combination immunotherapy as well as combinations of immunotherapy with targeted agents shown to significantly improve the outcomes of treatment-naïve mRCC patients (10-16). In mRCC, immunotherapy enhances adaptive immunity, granting the possibility for the immune system to recognize tumor antigens and to kill malignant cells (17). Activation of adaptive immunity against neoplastic antigens involves different receptors present on both malignant cells and immune cells, with inhibition or activation of signals (17). The tangled biological pathway underlying the role of anticancer immunity and its manipulation with therapies is not yet fully established. The immune compartment mainly includes T cells, natural-killer (NK) cells, B cells, macrophages and dendritic cells with complex interactions. T-cells are probably the major factor for both cellular and humoral immunological control of tumor growth. CD8+ T- cells are the main effectors of the anti-tumor immune response, recognizing antigens expressed by malignant cells and, once activated, killing neoplastic cells (18). CD4+ T-cells help in generating an immune response by stimulating CD8+ T-cells, macrophages and B-lymphocytes. The activation of effector and memory CD8+ T-cells occurs by the interaction with antigen-presenting cells (APC) via the T-cell receptor (TCR) and major histocompatibility complex (MHC)/peptide antigen (19). The most known ICls are the cytotoxic T-lymphocyte-associated antigen 4 (CTLA-4) pathway and the Programmed death 1 (PD-1) with its ligand (PD-L1) $(17,20)$. These two complexes are the targets of several drugs experimented in different clinical trials and now included in clinical practice. Anti CTLA-4 agents modulate the activation of T-cells binding to the CD80 and CD86 ligands, compromising CD28 for greater affinity (21). This results in a modulated activation of naive T-cells and memory. The success achieved by ipilimumab, a CTLA-4 inhibitor, in metastatic melanoma demonstrated that the inhibition of this immuno-checkpoint might stimulate the host immune system against neoplastic antigens, with tumor cells' death (22). However, in MRCC ipilimumab did not show a similar significative benefit. PD-1 is an inhibitory receptor expressed by activated T-cells, B-cells, monocytes, and natural killer (NK) cells. PD-L1 and PD-L2 are two wellknown ligands that activate PD-1. In particular, PD-L1 is expressed in different cells, including APC and malignant cells. The interplay of PD-1 with PD-L1 is responsible for the immunosuppressive effects of PD-1 (23) by inhibiting the proliferation, survival, and function of CD8+ lymphocytes, and promoting the differentiation of CD4+ T-cells into regulatory $T$ lymphocytes (Tregs). This mechanism can induce apoptosis of infiltrating tumor cells. Nonetheless, PD-L2 is responsible for the inhibition of T-cell activation (23). Anti-PD1 agents, such as pembrolizumab and nivolumab, and anti-PD-L1 drugs, such as durvalumab, atezolizumab and avelumab, led to a revolutionary approach in the therapeutic management of different solid neoplasms, including melanoma, non-small cell lung cancer, mRCC, urothelial carcinoma and Merkel cell carcinoma, but also in hematologic malignancies such as lymphomas $(10-15,24)$. This review summarizes current immunotherapeutic agents approved for mRCC.

\section{Combination therapy}

The frontline treatment of mRCC has been transformed since the approval of the immunotherapeutic combination. The two regulatory agency Food and Drug Administration (FDA) and European Medicines Agency (EMA) approved in 2018 the combination nivolumab plus ipilimumab in treatment-naïve mRCC patients at intermediate-poor risk and two other combinations in 2019 regardless of the risk category group: avelumab plus axitinib and pembrolizumab plus axitinib. 


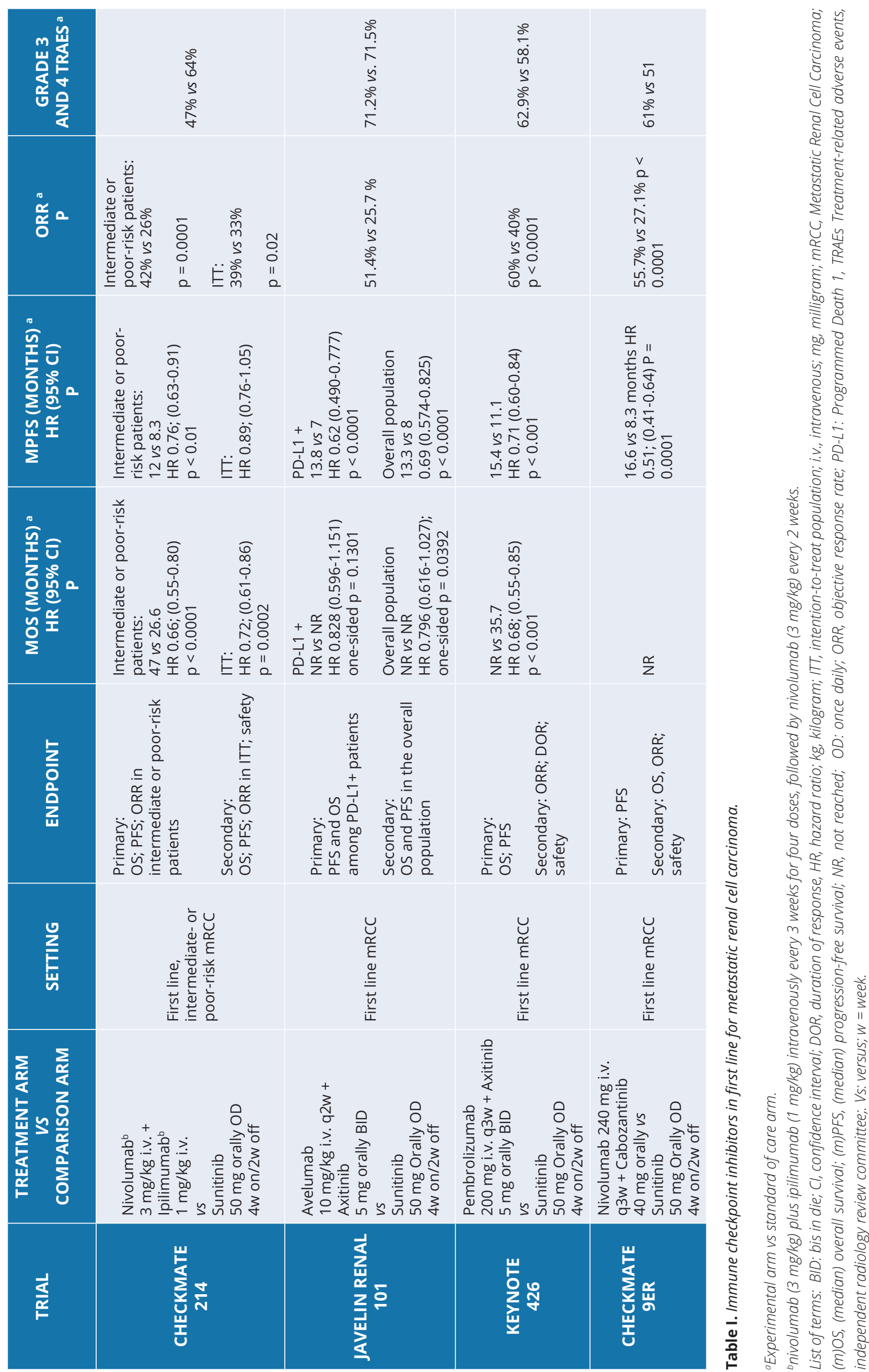


A.
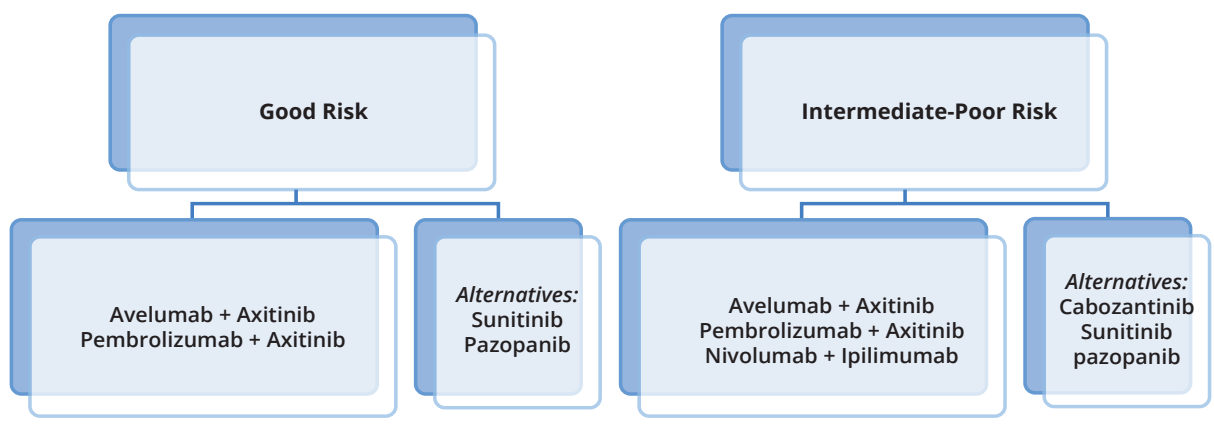

First line

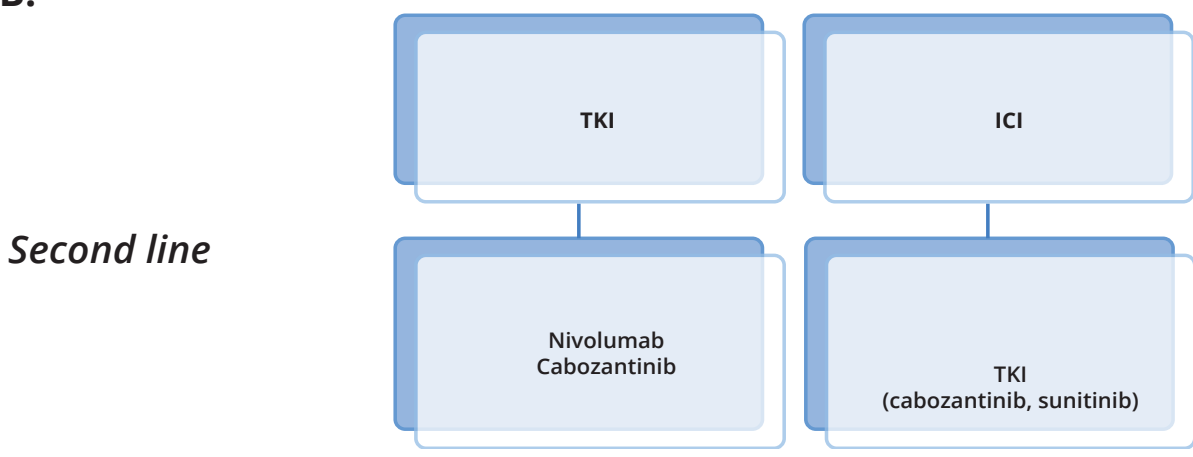

Figure 1. a. Summary of immunotherapy combinations approved by EMA and FDA for the first line treatment of metastatic renal cell carcinoma according to risk category group. TKI could also be an option; $\boldsymbol{b}$. Panel of options for the second lines after ICl or TKI. Abbreviations: TKI, tyrosine kinase inhibitor; ICI, Immune checkpoint inhibitor.

\section{Immune Checkpoint Inhibitors}

In the Checkmate 214 phase III trial 1096 treatment-naïve mRCC patients were randomly assigned to receive nivolumab $3 \mathrm{mg} / \mathrm{kg}$ plus ipilimumab $1 \mathrm{mg} / \mathrm{kg}$ every 3 weeks, followed by nivolumab at the same dose every 2 weeks versus sunitinib $50 \mathrm{mg}$ daily schedule 4 weeks on and 2 weeks off. Primary endpoints were objective response rate (ORR), progression-free survival (PFS) and overall survival (OS) in International mRCC Database Consortium (IMDC) intermediate-poor risk patients. Secondary endopoints were ORR, PFS and OS in any risk patients and safety in all treated patients. ORR, PFS and OS in IMDC favorable-risk were exploratory endpoints. At the first data cut-off, OS, PFS and ORR resulted significantly improved for the combination nivolumab plus ipilimumab versus sunitinib in intermediate-poor-risk patients (11). The significant superiority of the immunotherapeutic combination over sunitinib for intermediate-poor risk patients with $\mathrm{mRCC}$ was confirmed at the median follow-up of 42 months. In summary, long-term results showed the maintained benefit for the combination over sunitinib in terms of OS (47.0 versus 26.6 months, $\mathrm{HR}=0.66 ; 95 \% \mathrm{Cl}=0.55$ $0.80 ; \mathrm{P}<0.0001)$, $\mathrm{PFS}(12.0$ versus 8.3 months, $\mathrm{HR}=$
$0.76 ; 95 \% \mathrm{Cl}=0.63-0.91 ; \mathrm{P}<0.01)$ and ORR $(42.1 \%$ versus $26.3 \%$, respectively; $P=0.0001$ ) in intermediate-poor-risk mRCC patients. The exploratory analysis of the efficacy of the combination nivolumab plus ipilimumab compared to sunitinib in favorable-risk patients showed no benefit in OS (HR 1.19, $95 \% \mathrm{Cl} 0.77-1.85, \mathrm{P}=0.44$ ) and as expected, the median OS was not reached in either group. Duration of response was longer with nivolumab plus ipilimumab (HR, 0.46-0.54), and more patients achieved complete response (10.1\%-12.8\% vs $1.4 \%-5.6 \%$ ) regardless of risk group (12). Certain histologic features, as sarcomatoid histology, are associated with worse clinical outcomes. Up to $20 \%$ of mRCC patients present a sarcomatoid dedifferentiation. Since this subtype express high level of PD-1 and PD-L1, immunotherapy represents a promising therapy for these patients. A post-hoc analyses of Checkmate 214 was conducted focusing on intermediate-poor risk, advanced clear-cell RCC with sarcomatoid features. The descriptive analyses performed at a minimum follow-up of 30 months, confirmed promising efficacy in terms of ORR (56.7\% versus 19\%) and complete response rate (18.3\% versus 0$)$, OS (31.2 versus $13.6, \mathrm{HR} 0.55)$, and PFS (8.4 versus 4.9 months, $H R=0.61$ ) with 
nivolumab plus ipilimumab compared to suninitib in previously untreated, intermediate-poor risk, advanced clear-cell RCC with sarcomatoid features (25). In the primary analysis treatment-related adverse events (TRAE) occurred in $93 \%$ of patients treated with nivolumab plus ipilimumab and in $97 \%$ of patients treated with sunitinib. Grade 3 or 4 events occurred $46 \%$ and $63 \%$ of patients, respectively. TRAE events leading to discontinuation occurred in $22 \%$ and $12 \%$ of the patients in the respective groups. No new safety signals emerged to long-term follow-up. Concerning the issue of survival in patients who had to discontinue immunotherapy for TRAE, Tannir et al. conducted a posthoc analysis. The analysis showed that a benefit in OS persisted in patients despite having to discontinue therapy due to adverse events (26).

\section{Antiangiogenic and immunotherapy combinations} Immunotherapy was also combined with antiangiogenic treatment due to their synergistic effect. Immunosuppresion and angiogenesis represent the key mechanism in mRCC pathogenesis. The two mechanisms interact with each other determining changes in tumor microenvironment (TME). The complex interconnection between TME and immune system was exploited to enhance the immune responses obtained by ICls alone. The VEGFR signal blockade, in fact, leads to a modulation and recovery of TME and host immunity useful to enhance the anti-tumor immune response.
Combining immunotherapy with antiangiogenic treatment in fact, showed to improve outcomes in mRCC patients compared to TKI monotherapy. The KEYNOTE-426 trial is a large phase III trial that enrolled 861 patients to show a superiority of the combination of pembrolizumab (200 mg every 3 weeks for up to 35 cycles) plus axitinib (5 mg orally twice daily) over sunitinib (50 mg for 4 weeks in 6 -week cycles) in untreated mRCC patients. Initial data showed an improvement in OS $(90 \%$ versus $78 \%, \mathrm{HR}=0.53 ; 95 \% \mathrm{Cl}=0.38-0.74 ; \mathrm{P}<0.0001)$ and ORR ( $59 \%$ versus $36 \%, \mathrm{P}<0.001)$ with pembrolizumab plus axitinib across all the IMDC risk groups and regardless of PD-L1 expression (15). The updated data presented at the 2020 annual ASCO congress confirmed the superiority of the combination over sunitinib at the median follow-up of 23 months. Considering the intention-to-treat population, median OS was not yet reached for the patients assigned to receive the combination versus 35.7 months for the patients assigned to sunitinib $(\mathrm{HR}=0.68 ; 95 \% \mathrm{Cl}=0.55-0.85 \mathrm{P}<0.001)$. Median PFS was 15.4 months and 11.1 months in pembrolizumab plus axitinib arm and sunitinib arm respectively $(\mathrm{HR}=0.71 ; 95 \% \mathrm{Cl}=0.60-0.84, \mathrm{P}<0.001)$. In addition, the ORR was $60.2 \%$ with the combination and $40 \%$ in the sunitinib arm, with a complete response rate of $9 \%$ versus 3\%. Grouping patients by IMDC risk, significant differences in OS and PFS (HR of 0.63 for OS and 0.69 for PFS) were observed for patients with intermediate-poor risk disease,

\begin{tabular}{|c|c|c|c|c|c|}
\hline $\begin{array}{c}\text { TRIAL } \\
\text { CLINICALTRIALS.GOV }\end{array}$ & $\begin{array}{l}\text { PHASE AND } \\
\text { DESIGN }\end{array}$ & TREATMENT ARM & $\begin{array}{c}\text { COMPARISON } \\
\text { ARM }\end{array}$ & SETTING & $\begin{array}{l}\text { PRIMARY } \\
\text { ENDPOINT }\end{array}$ \\
\hline $\begin{array}{c}\text { CLEAR } \\
\text { NCT02811861 }\end{array}$ & $\begin{array}{l}\text { III, randomized, } \\
\text { open label }\end{array}$ & $\begin{array}{l}\text { Lenvatinib + } \\
\text { Everolimus or } \\
\text { Pembrolizumab }\end{array}$ & Sunitinib & $\begin{array}{l}\text { First line } \\
\text { mRCC }\end{array}$ & $\begin{array}{l}\text { PFS by } \\
\text { independent } \\
\text { review }\end{array}$ \\
\hline $\begin{array}{l}\text { COSMIC-313 } \\
\text { NCT03937219 }\end{array}$ & $\begin{array}{l}\text { III, randomized, } \\
\text { open label }\end{array}$ & $\begin{array}{l}\text { Cabozantinib } \\
+ \text { Nivolumab + } \\
\text { Ipilimumab }\end{array}$ & $\begin{array}{l}\text { Nivolumab + } \\
\text { Ipilimumab }\end{array}$ & $\begin{array}{l}\text { First line, } \\
\text { intermediate- } \\
\text { or poor-risk } \\
\text { mRCC }\end{array}$ & $\begin{array}{l}\text { PFS per blinded } \\
\text { independent } \\
\text { central review }\end{array}$ \\
\hline $\begin{array}{l}\text { PDIGREE STUDY } \\
\text { NCT03793166 }\end{array}$ & $\begin{array}{l}\text { III, randomized, } \\
\text { open label }\end{array}$ & $\begin{array}{l}\text { Cabozantinib + } \\
\text { Nivolumab }\end{array}$ & $\begin{array}{l}\text { Nivolumab + } \\
\text { Ipilimumab }\end{array}$ & $\begin{array}{l}\text { First line } \\
\text { mRCC }\end{array}$ & OS \\
\hline NCT03149822 & $\begin{array}{l}\text { I/II, open label, } \\
\text { single arm }\end{array}$ & $\begin{array}{l}\text { Pembrolizumab + } \\
\text { Cabozantinib }\end{array}$ & & $\begin{array}{l}\text { First or } \\
\text { second line } \\
\text { mRCC }\end{array}$ & ORR (CR + PR) \\
\hline NCT03200587 & Ib, open label & $\begin{array}{l}\text { Avelumab + } \\
\text { Cabozantinib }\end{array}$ & & $\begin{array}{l}\text { First line } \\
\text { mRCC }\end{array}$ & $\begin{array}{l}\text { DLTs, AEs, } \\
\text { RP2D }\end{array}$ \\
\hline
\end{tabular}

Table II. Ongoing clinical trials evaluating antiangiogenics and immune checkpoint inhibitors combinations in metastatic renal cell carcinoma. List of terms: AEs, adverse events; CR, complete response; DLT, dose limiting toxicity; mRCC, Metastatic Renal Cell Carcinoma; ORR, objective response rate; OS, overall survival; PFS, progression-free survival; PR partial response; RP2D, recommended Phase 2 dose. 
while no significant differences in OS or PFS were observed for patients with favorable-risk disease. However, the favorable-risk group had a better ORR (69.6 versus $50.4 \%$ and 55.8 versus $35.2 \%$, respectively) and a higher complete response rate (11\% versus $6 \%$ and 8 versus $2 \%$, respectively) with pembrolizumab plus axitinib versus sunitinib than did the intermediate-poor group (16).

In the JAVELIN phase III trial 442 treatment-naive mRCC patients were randomly assigned to receive a PD-L1 inhibitor, avelumab $(10 \mathrm{mg} / \mathrm{kg}$ intravenous infusion every 2 weeks) and a multikinase inhibitor, axitinib (5 mg orally twice daily) or sunitinib (50 mg orally once daily schedule 4 weeks on and 2 weeks off) (13). The two independent primary endpoints were PFS and OS among patients with PD-L1+ tumors (defined as $\geq 1 \%$ of immune cells staining positive within the tumor area of the tested tissue sample) (13). Key secondary endpoints were OS and PFS in the overall population. At a minimum follow-up of 13 months, among the patients with PD-L1-positive median PFS was 13.8 versus 7.0 months $(\mathrm{HR}=0.62,95 \% \mathrm{Cl}=0.490-0.777$; $\mathrm{P}<0.0001$ ) and ORR was $55.9 \%$ versus $27.2 \%$ with avelumab plus axitinib and sunitnib respectively (13). Considering the overall population, PFS was 13.3 versus 8.0 months in the avelumab plus axitinib and sunitinib arms, respectively ( $H R=0.69$, $95 \% \mathrm{Cl}=0.5740 .825$; one-sided $\mathrm{P}<0.0001$ ) (13). In conclusion, the combination showed to be superior in terms of PFS irrespective of IMDC risk group and PD-L1 expression. OS data were immature (13). TRAE occurred in $99.5 \%$ versus $99.3 \%$ pf patients treated with avelumab plus axitinib versus sunitinib respectively (13). Discussing safety data, the events were G3 or higher in $71.2 \%$ and $71.5 \%$ of the patients in the respective groups (13).

The combination nivolumab plus cabozantinib recently demonstrated to be very promising. The CheckMate-9ER tested the combination and results were presented very recently at the ESMO 2020 annual congress. The positive results support the increasing number of data showing that TKIs may create a more immune-permissive tumor microenvironment that could enhance the response to checkpoint. The combination was recently approved by FDA. In detail, CheckMate-9ER is the phase III trial, randomizing 651 previously untreated mRCC patients to receive nivolumab $240 \mathrm{mg}$ intravenous infusion every 2 weeks plus cabozantinib $40 \mathrm{mg}$ orally daily or sunitinib $50 \mathrm{mg}$ daily (schedule 4 weeks on and 2 weeks off). Primary endpoint was PFS. Secondary endpoint were OS, ORR and safety. The study met its primary endpoint showing a consistent benefit in terms of PFS for the combination of nivolumab plus cabozantinib over sunitinib (16.6 versus 8.3 months $\mathrm{HR}=$ $0.51 ; 95 \% \mathrm{Cl}=0.41-0.64, \mathrm{P}=0.0001)$. The superiority of nivolumab plus cabozantinib over sunitinib was observed also in terms of ORR $(55.7 \%$ versus $27.1 \%$ $p<0.0001$ ) with complete response rate of $8.0 \%$ versus $4.6 \%$. The benefit in PFS and ORR was demonstrated in numerous subgroups including age, sex, PD-L1 expression, bone metastases, and IMDC risk group. The combination was well tolered and reflected the known safety profiles of both nivolumab and cabozantinib. TRAE for any grade occurred in 97\% versus $93 \%$ of patients treated with nivolumab plus cabozantinib versus sunitinib respectively. The events were G3 or higher in $61 \%$ versus $51 \%$ of patients in the respective groups. Moreover, patients treated with nivolumab plus cabozantinib reported significantly better health-related quality of life than those treated with sunitinib at most time points, according to National Comprehensive Cancer Network-Functional Assessment of Cancer Therapy (NCCN-FACT) Kidney Symptom Index 19 (FKSI-19) scores. Longerterm data for OS are certainly needed because they are still immature (27).

All the studies mentioned above combining immunotherapeutic drugs or checkpoint inhibitors with a TKI included clear cells carcinoma and excluded rare histologies such as collecting duct carcinoma or papillary tumors

\section{DISCUSSION}

The approval of therapeutic combos as treatment for $\mathrm{mRCC}$ is rapidly changing the clinical practice. The rationale for combining different therapies was rooted in cancer-immunity cycle. The cycle consists into a series of functional stepwise events involving stimulatory and inhibitory factors to obtain an efficient control of cancer growth by the immune system (19). The synergistic effect that can result from the combination of two different therapies has been exploited to enhance the anti-tumor immune response obtained by ICls monotherapy (28). In detail, the CTLA-4 inhibition, which leads to an active immune response at the level of T-cell proliferation has a synergistic effect with PD-1 inhibition, which modulates the immune response at the level of the tumor bed (19). On the other hand, combining ICls with VEGF- or VEGFR-directed ther- 
apy significantly improved the outcomes of mRCC patients compared to TKI monotherapy. The VEGFR signal blockade, in fact, exert immunomodulatory activities, recovering TME and host immunity. This effect permitted to enhance the anti-tumor immune response obtained by ICls alone (28).

We are moving into a phase where different and effective therapies will be available, without knowing how to select patients. For this purpose, having predictive biomarkers to guide therapeutic decisions will be critical. Direct comparison of studies leading to approval of the combinations should be avoided $(11,13,15)$. However, we can derive useful information. Treatment choices could in fact be based on different clinical insights deriving from the characteristics of patients, of disease or drug activity. Concerning drug activities, we know that safety profile or timing of action is different for TKI and immunotherapy. In detail, ICls are associated with meaningful long-lasting responses (11). The CheckMate 214 trial confirmed the long-term benefit of the immunotherapy combination nivolumab plus ipilimum$a b$ in intermediate-poor risk at 42 months minimum follow-up. The study showed no separation in curves for the first months but a survival plateau occurs at 24-months, meaning that responses with the immune-combo are durable on the long-term compared to sunitinib, where response continues to decline (11). Compared to CheckMate 214, the follow-up with pembrolizumab plus axitinib is not so long yet. However, the OS curves separate since the beginning compared to sunitinib, meaning that the combination starts to work early. Therefore, we can derive that the combination ICIs-TKI could be preferred when shrinkage is needed for aggressive or rapidly progressive disease or for symptomatic patients (15). On the other hand, ipilimumab plus nivolumab or the combo cabozantinib plus nivolumab could be preferred if complete response is the prefixed objective, However, a high percentage of progressive disease as best response were observed in CheckMate 214 (11, 27, 29, 30).

Safety profile could also be helpful in clinical practice. In the CheckMate 214, 25\% of patients discontinued treatment due to TRAEs, the most occurring during the induction phase. In the Keynote 426, G3 or higher adverse events of any cause occurred in $66 \%$ and $62.4 \%$ of patients in the pembrolizumab plus axitinib group and in the sunitinib group, respectively. Moreover, axitinib is given full dose, different from the 9ER trial, where immunotherapy is combined to cabozantinib at reduced dose from the beginning $(11,15,27)$. Safety issue for TKI-immuno combo unfortunately, tend to persist over time due to prolonged administration of both agents. The possibility of using a TKI just for a limited period according to a possible intermittent schedule are under investigation with the aim to avoid overtreatment and reduce toxicity and costs. Ultimately, the quest for optimal methods to select patients may involve refinement of tissue-based analysis, angiogenesis and inflammatory gene expression signatures and blood-based biomarkers, among others $(31,32)$. The IMmotion150 trial investigating the combination with the angiogenesis inhibitor bevacizumab and the PD-L1 inhibitor atezolizumab showed different biologic subgroups based on gene expression (33). Effector T cells, interferon y or angiogenesis gene expression signatures, could be used to select patients more responsive to the immunotherapy combinations over TKIs and viceversa. Moreover, favorable risk patients showed a more angiogenic phenotype, suggestsing that VEGF agents could remain a good treatment choice (33). Question about the optimal sequencing remains open and prospective trial investigating innovative therapies are needed. Patients rapidly progress on therapy, so improved therapeutic option with superior efficacy are needed and enrollement into clinical trials investigating promising therapies with new mechanism of action is recommended.

\section{CONCLUSIONS}

The treatment landscape of $\mathrm{mRCC}$ is rapidly evolving, but several unmet needs remain. We are moving into a phase where different therapies are available, without knowing how to select patients. Certainly, the identification of predictive biomarker to guide therapeutic decisions is essential. Going forward, tissue-based analysis, whole genome sequencing and epigenetic analysis will probably help to understand the biology of RCC and to distinguish genomic signatures that can predict response to different treatment strategies.

\section{ETHICS}

\section{Fundings}

There were no institutional or private fundings for this article. 


\section{Conflict of interests}

The authors have declared no conflict of interests.

\section{Availability of data and material}

All the data supporting the findings of this study are available within the article and can be shared just before a reasonable request to the corresponding author.

\section{Authors' contribution}

All the authors contributed equally to conception, data collection, analysis and writing of this paper.

\section{REFERENCES}

1. Capitanio U, Montorsi F. Renal cancer. Lancet 2016;387:894-906.

2. Escudier B, Eisen T, Stadler WM, et al. Sorafenib in advanced clear-cell renal-cell carcinoma. $\mathrm{N}$ Engl J Med 2007;356:125-4.

3. Motzer RJ, Hutson TE, Tomczak P, et al. Sunitinib versus interferon alfa in metastatic renal-cell carcinoma. N Engl J Med 2007;356:115-24.

4. Rini BI, Halabi S, Rosenberg JE, et al. Phase III trial of bevacizumab plus interferon alfa versus interferon alfa monotherapy in patients with metastatic renal cell carcinoma: final results of CALGB 90206.J Clin Oncol 2010;28(13):2137-43.

5. Sternberg CN, Davis ID, Mardiak J, et al. Pazopanib in locally advanced or metastatic renal cell carcinoma: results of a randomized phase III trial. J Clin Oncol 2010;28:1061-8.

6. Choueiri TK, Escudier B, Powles T, et al. Cabozantinib versus everolimus in advanced renal-cell carcinoma. N Engl J Med 2015;373:1814-23.

7. Motzer RJ, Escudier B, Oudard S, et al. Phase 3 trial of everolimus for metastatic renal cell carcinoma: final results and analysis of prognostic factors. Cancer 2010;116:4256-65.

8. Choueiri TK, Hessel C, Halabi S, Sanford B, Michaelson MD, Hahn O, et al. Cabozantinib versus sunitinib as initial therapy for metastatic renal cell carcinoma of intermediate or poor risk (Alliance A031203 CABOSUN randomised trial): Progression-free survival by independent review and overall survival update. Eur J Cancer 2018;94:115-125. Erratum in: Eur J Cancer 2018;103:287.

9. George DJ, Hessel C, Halabi S, et al. Cabozantinib versus sunitinib for untreated patients with advanced renal cell carcinoma of intermediate or poor risk: subgroup analysis of the alliance A031203 CABOSUN trial. Oncologist 2019;24(11):1497-1501.

10. Motzer RJ, Tykodi SS, Escudier B, et al. Final analysis of the CheckMate 025 trial comparing nivolumab (NIVO) versus everolimus (EVE)

with $>5$ years of follow-up in patients with advanced renal cell carcinoma (aRCC). J Clin Oncol 2020;38(supplᄀ_6):617.

11. Motzer RJ, Rini BI, McDermott DF, et al. Nivolumab plus ipilimumab versus sunitinib in first-line treatment for advanced renal cell carcinoma: extended follow-up of efficacy and safety results from a randomized, controlled phase 3 trial. Lancet Oncol 2019;20(10):1370-85.

12. Tannir NM, McDermott DF, Escudier B, et al. Overall survival and independent review of response in CheckMate 214 with 42-month follow-up: first-line nivolumab + ipilimumab $(\mathrm{N}+\mathrm{I})$ versus sunitinib (S) in patients (pts) with advanced renal cell carcinoma (aRCC). J Clin Oncol 2020;38(6_suppl):609.

13. Choueiri TK, Motzer RJ, Rini BI, et al. Updated efficacy results from the JAVELIN Renal 101 trial: first-line avelumab plus axitinib versus sunitinib in patients with advanced renal cell carcinoma. Ann Oncol 2020;31(8):1030-9.

14. Rini BI, Powles T, Atkins MB, et al. Atezolizumab plus bevacizumab versus sunitinib in patients with previously untreated metastatic renal cell carcinoma (IMmotion151): a multicentre, open-label, phase 3, randomised controlled trial. Lancet 2019;393(10189):2404-15.

15. Rini BI, Plimack ER, Stus V, et al. Pembrolizumab plus axitinib versus sunitinib for advanced renal-cell carcinoma. $N$ Engl J Med 2019;380(12):1116-27.

16. Plimack ER, Rini BI, Stus V, et al. Pembrolizumab plus axitinib versus sunitinib as first-line therapy for advanced renal cell carcinoma (RCC): updated analysis of KEYNOTE-426. J Clin Oncol 2020;38(15_suppl):5001.

17. Zhang S, Zhang E, Long J, et al. Immune infiltration in renal cell carcinoma. Cancer Sci 2019;110(5):1564-72.

18. Miller JF, Sadelain M. The journey from discoveries in fundamental immunology to cancer immunotherapy. Cancer Cell 2015;27(4):439-49. 
19. Chen DS, Mellman I. Oncology meets immunology: the cancer-immunity cycle. Immunity 2013;39(1):1-10.

20. Kwon B. Is CD137 ligand (CD137L) signaling a fine tuner of immune responses? Immune Netw 2015;15(3):121-4.

21. Seidel JA, Otsuka A, Kabashima K. Anti-PD-1 and anti-CTLA-4 therapies in cancer: mechanisms of action, efficacy, and limitations. Front Oncol 2018;8:86.

22. Hodi FS, O'Day SJ, McDermott DF, et al. Improved survival with ipilimumab in patients with metastatic melanoma. [Published correction appears in N Engl J Med 2010;363(13):1290]. N Engl J Med 2010;363(8):711-23.

23. Marinelli O, Annibali D, Aguzzi C, et al. The controversial role of PD-1 and its ligands in gynecological malignancies. Front Oncol 2019;9:1073.

24. Chevrier S, Levine JH, Zanotelli VRT, et al. An immune atlas of clear cell renal cell carcinoma. Cell 2017;169:736-49.

25. McDermott DF, Choueiri TK, Motzer RJ, Arén Frontera O, et al. CheckMate 214 Post Hoc Analyses of Nivolumab Plus Ipilimumab or Sunitinib in IMDC Intermediate/Poor-Risk Patients With Previously Untreated Advanced Renal Cell Carcinoma With Sarcomatoid Features. J Clin Oncol 2019;37(15_suppl):4513.

26. Tannir NM, McDermott DF, Escudier B, Hammers $\mathrm{HJ}$, et al. Overall survival and independent review of response in CheckMate 214 with 42-month follow-up: First-line nivolumab + ipilimumab $(\mathrm{N}+\mathrm{l})$ versus sunitinib $(\mathrm{S})$ in patients (pts) with advanced renal cell carcinoma (aRCC). J Clin Oncol 2020;38(6_suppl):609.
27. Choueiri TK, Powles T, Burotto $M$, et al. Nivolumab + cabozantinib vs sunitinib in firstline treatment for advanced renal cell carcinoma: first results from the randomized phase 3 CheckMate 9ER trial. Ann Oncol 2020;31(suppl_4):S1142-S1215.

28. Mennitto A, Huber V, Ratta R, et al. Angiogenesis and immunity in renal carcinoma: can we turn an unhappy relationship into a happy marriage? J Clin Med 2020;9(4):930.

29. Sepe P, Mennitto A, Corti F, Procopio G. Immunotherapeutic Targets and Therapy for Renal Cell Carcinoma. Immunotargets Ther 2020;9:273-88.

30. Procopio G, Sepe P, Claps M, de Braud F, Verzoni E. Should we use combination therapy for all advanced renal cell carcinoma? Lancet Oncol 2019;20(10):1331-2.

31. Raimondi A, Sepe P, Claps M, Verzoni E, Procopio G. Do biomarkers play a predictive role for response to novel immunotherapeutic agents in metastatic renal cell carcinoma? Expert Opin Biol Ther 2019;19(11):1107-10.

32. Raimondi A, Randon G, Sepe $P$, et al. The evaluation of response to immunotherapy in metastatic renal cell carcinoma: open challenges in the clinical practice. Int J Mol Sci 2019;20(17):4263.

33. McDermott DF, Huseni MA, Atkins MB, et al. Clinical activity and molecular correlates of response to atezolizumab alone or in combination with bevacizumab versus sunitinib in renal cell carcinoma. [Published correction appears in Nat Med. 2018;24(12):1941]. Nat Med 2018;24(6):749-57. 